\title{
Photorefractive polymeric optical spatial solitons
}

\author{
Ming-Feng Shih and Fang-Wen Sheu \\ Department of Physics, National Taiwan University, Taipei 10764, Taiwan
}

Received August 2, 1999

\begin{abstract}
We predict the formation of optical spatial solitons in photorefractive polymers. The orientational enhancement from the doped chromophores and the dependency of the quantum efficiency of generating mobile holes on the electric field make the polymeric solitons behave differently from other photorefractive solitons. (C) 1999 Optical Society of America
\end{abstract}

OCIS codes: $190.5530,250.5460,160.5320$.

Since photorefractive (PR) optical spatial solitons were discovered $^{1}$ in PR inorganic crystals, many types of PR soliton and interesting soliton phenomena have been discovered and studied. ${ }^{2}$ The PR screening soliton ${ }^{3-5}$ is one of the most thoroughly studied solitons. It occurs when an externally biasing electric field is partially screened by space charges induced by a soliton light beam. The total biasing and space-charge field can, by means of the electro-optic effect, create a waveguidelike refractive-index profile that focuses the soliton light beam. The formation of a PR screening soliton is a net result of the balance between the natural diffraction of the soliton light beam and the PR focusing effect.

PR polymers ${ }^{6-8}$ were discovered a year earlier than PR optical spatial solitons and have attracted much research interest. Of all PR polymers, guest-host PR polymers, ${ }^{9}$ made from photoconducting polymer hosts and doped with high levels of chromophores, are one of the most successful types of PR polymer. They provide high $P R$ efficiency because of their orientational enhancement,${ }^{10}$ which results from the rotational mobility of chromophores. In some systems, the orientational enhancement may contribute more than $88 \%$ of the index change to the PR effect. ${ }^{11}$ Except for the origin of the index change, the guest-host PR polymers operate very similarly to the PR inorganic crystals: Charges are excited by light, redistributed by a diffusion or biasing field, trapped, and yield a spacecharge field. The space-charge field then gives rise to the index change by means of an orientational and (or) an electro-optic effect. We therefore expect that PR polymers will be able to support PR solitons, and we name them PR polymeric solitons. In this Letter we examine intuitively and analytically how polymeric solitons can form. We find that the orientational enhancement and the dependency of the quantum efficiency of generating mobile holes on the electric field are the key factors that affect the polymeric solitons.

Assume that the polymer is arranged as in Fig. 1(a). Biasing field $E_{\text {ext }}$ is applied along $\hat{x}$, and the onedimensional soliton light beam is launched along $\hat{z}$. Before $E_{\text {ext }}$ is applied, thermal agitation causes the chromophores to be randomly oriented, giving rise to a homogeneous and isotropic refractive index. As $E_{\text {ext }}$ is applied, the birefringent chromophores align more along $\hat{x}$ [Fig. 1(d)]. This alignment yields a positive (negative) index change for an $\hat{x}-(\hat{y}-)$ polarized light [Figs. 1(e) and 1(f), dashed lines] for chromophores with its polarizability $\left(\alpha_{/ /}\right)$parallel to its dipole moment larger than its perpendicular polarizability $\left(\alpha_{\perp}\right)$. When a bright soliton light beam with intensity $I$ is launched [Fig. 1(b)], hole density ${ }^{9} \rho$ increases as $\phi\left(I+I_{b}\right)$, where $\phi$ is the quantum efficiency that depends on the static electric field and $I_{b}$ is a uniformly launched background illumination. If $E_{\text {ext }}$ is high enough, the diffusion effect ${ }^{9,12}$ is negligible. Therefore the holes travel in $\hat{x}$ and leave negatively ionized charge generators behind in the region where they are excited. The holes are retrapped after traveling some short distances. The separated positive and negative space charges induce electric field $E_{\mathrm{sc}}$ in $-\hat{x}$. This charge separation process keeps going on until steady state is reached, that is, when $E_{\mathrm{sc}}$ is accumulated to be large enough to balance $E_{\text {ext }}$. As a result, the total field $E=E_{\mathrm{sc}}+E_{\text {ext }}$ reaches its lowest value at the soliton peak and is a constant away from the soliton [Fig. 1(c)]. For polymers with glass-transition temperatures lower than the ambient temperature, the chromophores near the soliton peak can relax back [Fig. 1(d), central part] to be more randomly oriented and reduce the index change. The $\hat{x}-(\hat{y}-)$ polarized light thus sees an antiwaveguide (waveguidelike) index profile [Figs. 1(e) and 1(f), solid curves]. This means that the biased polymer is a self-defocusing (self-focusing) medium for an $\hat{x}-(\hat{y}-)$ polarized light beam. If the $\hat{y}$-polarized light beam is launched as the guided mode of the induced waveguide, it can self-trap without diffraction and become (a)

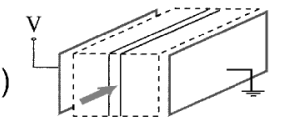

(b)

(c)

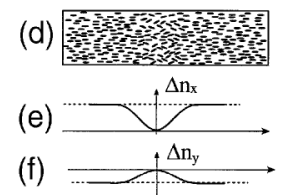

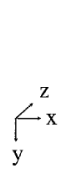

y

(g)
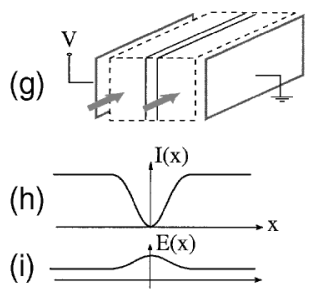

(j)

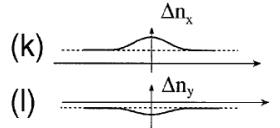

Fig. 1. Arrangement for observing (a) bright and (g) dark polymeric solitons. Formation of $(b-f)$ bright and $(h-l)$ dark polymeric solitons. (d), and (j) Alignment of the chromophores. The degrees of the alignment are exaggerated and should be more random in a real situation. 
an optical spatial soliton. By similar arguments, a dark soliton can be supported by the poled polymer if it is $\hat{x}$ polarized. This property, that polarization of the soliton decides whether bright or dark solitons can be supported, has been found in a cubic PR inorganic crystal. ${ }^{13}$ However, an opposite biasing field on a PR polymer does not reverse the sign of the index change.

In the analysis, we start from the model equations ${ }^{14}$ at steady state and the Helmholtz equation that describes the soliton light beam in $(1+1)$ dimensions:

$$
\begin{aligned}
J & =e \mu \rho E-e D \frac{\partial \rho}{\partial x}=\text { const. or } \quad \frac{\partial J}{\partial x}=0, \\
\frac{\partial N^{-}}{\partial t} & =s \phi\left(N-N^{-}\right)\left(I+I_{b}\right)-\frac{e \mu}{\epsilon_{0} \epsilon_{r}} \rho N^{-}=0, \\
\frac{\partial T^{+}}{\partial t} & =\frac{e \mu}{\epsilon_{0} \epsilon_{r}} \rho\left(T-T^{+}\right)-r T^{+}=0 \\
\frac{\partial E}{\partial x} & =\frac{e}{\epsilon_{0} \epsilon_{r}}\left(\rho-N^{-}+T^{+}\right) \\
& \left(\frac{\partial^{2}}{\partial x^{2}}+\frac{\partial^{2}}{\partial z^{2}}\right) E_{\mathrm{opt}}+\left(k_{0} n\right)^{2} E_{\mathrm{opt}}=0
\end{aligned}
$$

In Eqs. (1)-(4), $J$ is the current density, $e$ is the charge on a hole, $\mu$ is the hole mobility, $D$ is the diffusion coefficient, $N$ and $N^{-}$are the densities of total and ionized charge-generating molecules, respectively, $s$ is the photoexcitation cross section, $T$ and $T^{+}$are the densities of total and ionized charge traps, respectively, $r$ is the thermal detrapping rate, and $\epsilon_{r}$ is the static relative permittivity of the polymers. In Eq. (5), $n$ is the nonlinear refractive index to be determined and $E_{\text {opt }}(x, z)=\varphi(x, z) \exp \left(i k_{0} n_{b} z\right) \sqrt{I_{b}}+$ c.c. is the electric-field component, polarized in $\hat{x}$ (or $\hat{y}$ ) for a dark (or bright) soliton, of the optical beam of the soliton, where $\varphi$ is the amplitude of the slowly varying envelope normalized to $\sqrt{I_{b}}, n_{b}$ is the unperturbed index, $k_{0}=2 \pi / \lambda$, and $\lambda$ is the free-space wavelength of the light. The intensity of the soliton is $I=|\varphi|^{2} I_{b}$. The soliton intensity ratio is defined as the maximum of $|\varphi|^{2}$. The rate equation for the hole density is not listed because it is redundant with Eqs. (1)-(3). Note that three properties of PR polymers deserve attention: (1) by Onsager theory, ${ }^{15} \phi \propto E^{m}$ for $E$ from 10 to $100 \mathrm{~V} / \mu \mathrm{m}$, where $m$ is a material parameter that ranges from less than 1.0 to greater than 3.0; (2) mobility $^{16} \mu \propto \exp [C(\sqrt{E}-1)]$, where $C$ is an experimentally determined constant; and (3) by the Langevin model, ${ }^{9}$ the recombination and trapping rates in Eqs. (2) and (3) are $e \mu / \epsilon_{0} \epsilon_{r}$.

First we derive $\rho \approx\left(\epsilon_{0} \epsilon_{r} / e \mu\right)\left[s \phi N\left(I+I_{b}\right) / T^{+}\right][1-$ $\left.\left(\epsilon_{0} \epsilon_{r} / e T^{+}\right)(\partial E / \partial x)\right]^{-1}$ by steps similar to those in Ref. 4 to simplify Eqs. (2)-(4) with typical polymer parameters, ${ }^{14,17}$ of which $T^{+}$and $N^{-}$of the order of $10^{17} \mathrm{~cm}^{-3}$ and $N$ of the order of $10^{19} \mathrm{~cm}^{-3}$ are much larger than $\rho$, which is of the order of $10^{15} \mathrm{~cm}^{-3}$ under the soliton and background illumination of intensity $0.1 \mathrm{~W} / \mathrm{cm}^{2}$. Further, we drop ${ }^{18}$ the term that contains $\partial E / \partial x$ and the diffusion in Eq. (1) and obtain $\rho \approx$ $\left(\epsilon_{0} \epsilon_{r} s N / e T^{+}\right)\left[\phi\left(I+I_{b}\right) / \mu\right]$ and $J \approx e \mu \rho E$. Using the relationship $\phi \propto E^{m}$ and the constancy of $J$, we can easily derive

$$
E^{m+1}=E_{0}^{m+1}\left(I_{0}+I_{b}\right) /\left(I+I_{b}\right),
$$

in which the dependency on mobility disappears. The constants $E_{0}$ and $I_{0}$ are, respectively, electric field $E$ and soliton intensity $I$, far away from the soliton center.

We then turn to Eq. (5), which leads to the paraxial equation

$$
i \varphi_{z}+\frac{1}{2 k} \varphi_{x x}+\frac{k_{0}^{2}}{2 k} \Delta\left(n^{2}\right) \varphi=0,
$$

where $k=k_{0} n_{b}, \Delta\left(n^{2}\right)=n^{2}-n_{b}^{2}, \varphi_{z}=\partial \varphi / \partial z$, etc. Considering only the birefringence that is due to the orientational effect, ${ }^{11,12}$ which, e.g., accounts for more than $88 \%$ of the PR effect in polymers doped with 2,5-dimethyl-4-( $p$-nitrophenylazo)anisole or 99\% with $\mathrm{N}$-2-butyl-2, 6-dimethyl-4H-pyridone-4-ylidenecyanomethyl-2 acetate, we have the index change governed ${ }^{10,19}$ by $\Delta\left(n^{2}\right)_{x}=4 \pi N_{\mathrm{ch}} \Delta \alpha\left(\left\langle\cos ^{2} \theta\right\rangle-1 / 3\right)$ and $\Delta\left(n^{2}\right)_{y}=-\Delta\left(n^{2}\right)_{x} / 2$ for $x$ - and $y$-polarized light, respectively, where $\Delta \alpha=\alpha_{/ /}-\alpha_{\perp}$ and $N_{\text {ch }}$ is the density of chromophores. The distribution $\left\langle\cos ^{2} \theta\right\rangle=\int_{0}^{\pi} \exp \times$ $\left(-U / k_{B} T_{a}\right) \cos ^{2} \theta \sin \theta \mathrm{d} \theta / \int_{0}^{\pi} \exp \left(-U / k_{B} T_{a}\right) \sin \theta \mathrm{d} \theta$ is a function of dipole interaction energy $U=-\boldsymbol{\mu}_{D}$. $\mathbf{E}-\mathbf{p} \cdot \mathbf{E} / 2$ and ambient temperature $T_{a}$, where $\theta$ is the angle between the permanent dipole moment $\left(\boldsymbol{\mu}_{D}\right)$ and the electric field $(\mathbf{E})$ and $k_{B}$ is the Boltzmann constant. We neglect ${ }^{20}$ the induced dipole energy, $\mathbf{p} \cdot \mathbf{E} / 2$, which contributes little to $U$. The exponents in both integrands of $\left\langle\cos ^{2} \theta\right\rangle$ then become $\mu_{D} E_{T} \cos \theta / k_{B} T_{a}$. Equation (7), which contains $\left\langle\cos ^{2} \theta\right\rangle$ and with $E$ to be determined by Eq. (6), is a highly nonlinear differential-integral equation, which can be solved only numerically. However, $\left(\left\langle\cos ^{2} \theta\right\rangle-1 / 3\right)$, shown in Fig. 2, can be approximated $^{10}$ by the first nonzero term $0.043\left(\mu_{D} E / k_{B} T_{a}\right)^{2}$ of its power series for $\mu_{D} E / k_{D} T_{a}<0.8$ (Fig. 2, inset), which is typical of most PR guest-host polymers with $\mu_{D}<10 D$ for $E<100 \mathrm{~V} / \mu \mathrm{m}$ and $T_{a}=$ $300 \mathrm{~K}$. Equation (7) is simplified to

$$
i \varphi_{z}+\frac{1}{2 k} \varphi_{x x}+\frac{k_{0}^{2}}{2 k} C_{x, y} E^{2} \varphi=0
$$

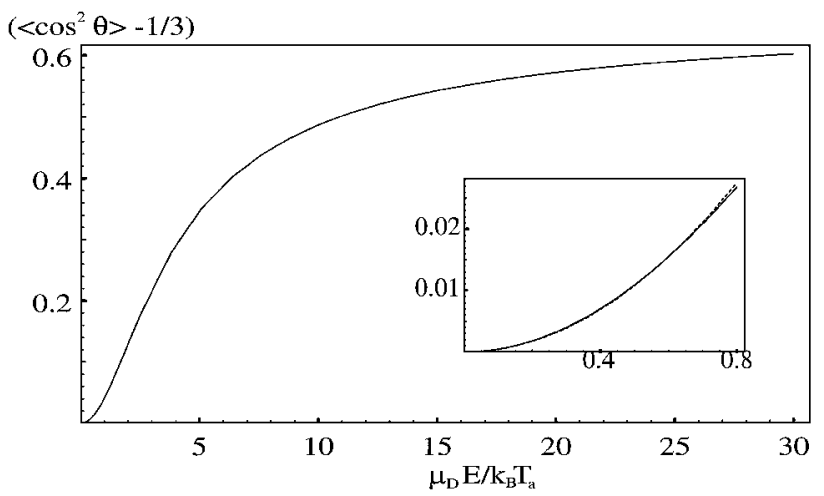

Fig. 2. Angle distribution $\left\langle\cos ^{2} \theta\right\rangle-1 / 3$. The inset shows that $\left\langle\cos ^{2} \theta\right\rangle-1 / 3$ (solid curve) can be approximated by $0.043\left(\mu_{D} E / k_{B} T_{a}\right)^{2}$ (dashed curve) for $\mu_{D} E / k_{B} T_{a}<0.8$. 

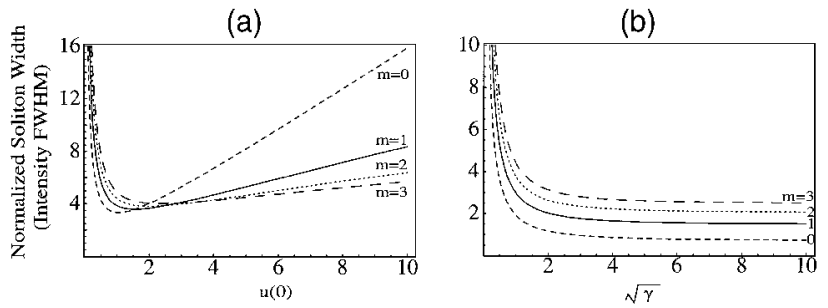

Fig. 3. Normalized soliton widths of (a) bright solitons as function of $u(0)$ and (b) dark solitons as a function of $\sqrt{\gamma}$ for several $m$ values.

with $C_{x}=0.54 N_{\mathrm{ch}} \Delta \alpha\left(\mu_{D} / k_{B} T_{a}\right)^{2}$ and $C_{y}=-C_{x} / 2$ for $x$ - and $y$-polarized light, respectively.

We can establish the envelope evolution equation by inserting Eq. (6) into Eq. (8):

$$
i \varphi_{\zeta}+\varphi_{\xi \xi}+\beta\left(\frac{\gamma+1}{|\varphi|^{2}+1}\right)^{2 /(m+1)} \varphi=0
$$

where $x_{0}$ is an arbitrary spatial width, $\zeta=z / 2 k x_{0}^{2}$, $\xi=x / x_{0}, \beta=k_{0}^{2} x_{0}^{2} C_{x, y} E_{0}^{2}$, and $\gamma=I_{0} / I_{b} . \quad \gamma$ is the intensity ratio for dark solitons, and $\gamma=0$ for bright solitons. Choosing $x_{0}=1 /\left(k_{0} E_{0} \sqrt{\left|C_{x, y}\right|}\right)$ to make $|\beta|=1$, we obtain the equation for the soliton envelope:

$$
\frac{d^{2} u}{d \xi^{2}}=\left[\Gamma \pm\left(\frac{\gamma+1}{u^{2}+1}\right)^{2 /(m+1)}\right] u,
$$

where the plus (minus) corresponds to $C_{y}\left(C_{x}\right)$ and bright (dark) solitons, $\varphi(\xi, \zeta)=u(\xi) \exp (i \Gamma \zeta), u$ is the normalized soliton envelope amplitude, and $\Gamma$ is the normalized soliton propagation constant. Equation (10) is actually the same as those for the PR screening solitons ${ }^{3,4}$ in noncentrosymmetric and centrosymmetric PR crystals $^{21}$ for $m=1$ and $m=0$, respectively. Equation (10) is solved by quadrature similar to that in Ref. 3 . For bright solitons, with initial conditions $u(0)=u_{0}, u^{\prime}(0)=0 ; u(\infty)=u^{\prime}(\infty)=$ $u^{\prime \prime}(\infty)=0$; and $u^{\prime \prime}(0) / u(0)<0$, we have $\Gamma=[(m+1) /$ $(m-1)]\left[1-\left(u_{0}^{2}+1\right)^{(m-1) /(m+1)}\right] / u_{0}^{2}$ for $m \neq 1$ and $\Gamma=-\ln \left(u_{0}^{2}+1\right) / u_{0}^{2}$ for $m=1$, where $u_{0}^{2}$ is the soliton peak intensity divided by $I_{b}$ or is called the bright soliton intensity ratio. For dark solitons, with $u^{2}(\infty)=\gamma, u^{\prime}(\infty)=u^{\prime \prime}(\infty)=0 ; u(0)=0$; and $u^{\prime}(0)$ being real, we have $\Gamma$ always equal to 1 . We can easily obtain the soliton profiles by putting $\Gamma$ into Eq. (10) and solving it numerically. We can also evaluate the required voltage across the polymer by integrating Eq. (6) after Eq. (10) is solved. The soliton widths, defined as the full width at half-maximum of the soliton intensity is normalized to $x_{0}$, are plotted in Fig. 3. To estimate the obtainable minimal soliton width we use the following PR polymer parameters $^{6,11}: \quad m=2.0, n_{b} \approx 1.6$, and $\Delta n_{x} \approx 1.3 \times 10^{-2}$ for $E_{\text {ext }}=100 \mathrm{~V} / \mu \mathrm{m}$ with $\lambda=700 \mathrm{~nm}$. Solitons of widths less than $1.5 \mu \mathrm{m}$ (dark solitons) or $3 \mu \mathrm{m}$ (bright solitons) are possible if the intensity ratio is adjusted to correspond to each minimal normalized soliton width. Nevertheless, for solitons of this size, the validity of the paraxial approximation and the validity of dropping the diffusion and $\partial E / \partial x$ terms need to be reinvestigated; such an investigation is beyond the scope of this Letter.

In conclusion, we have shown intuitively and analytically that photorefractive polymers may support PR polymeric optical spatial solitons.

This research is supported by the National Science Council, Taiwan, under contract NSC-89-2113-M002-034. M.-F. Shih's e-mail address is mfshih@phys. ntu.edu.tw.

\section{References}

1. M. Segev, B. Crosignani, A. Yariv, and B. Fischer, Phys. Rev. Lett. 68, 923 (1992).

2. M. Segev and G. Stegeman, Phys. Today 51(8), 42 (1998), and references therein.

3. M. Segev, G. C. Valley, B. Crosignani, P. Diporto, and A. Yariv, Phys. Rev. Lett. 73, 3211 (1994); M. Segev, M. Shih, and G. C. Valley, J. Opt. Soc. Am. B 13, 706 (1996).

4. D. N. Christodoulides and M. Carvalho, J. Opt. Soc. Am. B 12, 1628 (1995).

5. M.-F. Shih, M. Segev, G. C. Valley, G. Salamo, B. Crosignani, and P. DiPorto, Electron. Lett. 31, 826 (1995).

6. S. Ducharme, J. C. Scott, R. J. Twieg, and W. E. Moerner, Phys. Rev. Lett. 66, 1846 (1991).

7. K. Meerholz, B. Volodin, Sandalphon, B. Kippelen, and N. Peyghambarian, Nature 371, 497 (1994); M. Liphardt, A. Goonesekera, B. Jones, S. Ducharme, J. Takacs, and L. Zhang, Science 263, 367 (1994); A. Grunnet-Jepsen, C. Thompson, and W. Moerner, Science 277, 549 (1997).

8. C. Poga, P. Lundquist, V. Lee, R. Shelby, R. Twieg, and D. Burland, Appl. Phys. Lett. 69, 1047 (1996).

9. W. E. Moerner and S. M. Silence, Chem. Rev. 94, 127 (1994).

10. W. Moerner, S. Silence, F. Hache, and G. Bjorklund, J. Opt. Soc. Am. B 11, 320 (1994).

11. C. Moylan, R. Wortmann, R. Twieg, and I. McComb, J. Opt. Soc. Am. B 15, 929 (1998).

12. S. Schloter, U. Hofmann, P. Strohriegl, H. Schmidt, and D. Haarer, J. Opt. Soc. Am. B 15, 2473 (1998).

13. W. Krolikowski, N. Akhmediev, and B. Luther-Davies, Opt. Lett. 21, 782 (1996).

14. J. S. Schildkraut and A. V. Buettner, J. Appl. Phys. 72, 1888 (1992).

15. P. J. Melz, J. Chem. Phys. 57, 1694 (1972).

16. J. X. Mack, L. B. Schein, and A. Peled, Phys. Rev. B 39, 7500 (1989).

17. A. Grunnet-Jepsen, C. L. Thompson, R. J. Twieg, and W. E. Moerner, Appl. Phys. Lett. 70, 1515 (1997).

18. Both approximations were justified physically ${ }^{4,17}$ in terms of the inequality $E_{d} \ll E \ll E_{q}$, where $E_{q}$ and $E_{d}$ are the limiting space-charge field and the diffusion field, respectively, evaluated at a soliton width of not less than $5 \mu \mathrm{m}$.

19. J. W. Wu, J. Opt. Soc. Am. B 8, 142 (1991).

20. For a typical example from Ref. 19 , with $\mu_{D} \cong 10 \mathrm{D}$, $\Delta \alpha^{0}=5 \times 10^{-23} \mathrm{~cm}^{3}$, and $E=100 \mathrm{~V} / \mu \mathrm{m}$, the induced dipole contributes only one sixtieth of the total dipole energy.

21. M. Segev and A. J. Agranat, Opt. Lett. 22, 1299 (1997). 